\title{
Dirichlet-Neumann preconditioning for stabilised unfitted discretization of high contrast problems
}

\author{
B. Ayuso de Dios, K. Dunn, M. Sarkis, and S Scacchi
}

\begin{abstract}
We present a Dirichlet-Neumann preconditioner for a Nitsche stabilised unfitted method for high-contrast interface elliptic problems. We demonstrate numerical optimality and robustness of the solver in the soft and hard inclusion cases.
\end{abstract}

\section{Introduction}

Let $\Omega \subset \mathbb{R}^{2}$ be a polygonal domain with an immersed simple closed smooth interface $\Gamma \in \mathscr{C}^{2}$, such that $\bar{\Omega}=\bar{\Omega}^{-} \cup \bar{\Omega}^{+}$, and $\Gamma:=\bar{\Omega}^{-} \cap \bar{\Omega}^{+}$is far away from $\partial \Omega$ (i.e, either $\Omega^{+}$or $\Omega^{-}$is a floating subdomain; i.e., one of them does not touch $\partial \Omega$ ). Given $f \in L^{2}(\Omega)$ we set $f^{ \pm}=f_{\left.\right|_{\Omega^{ \pm}}}$and consider the problem of finding $u_{*}$ such that

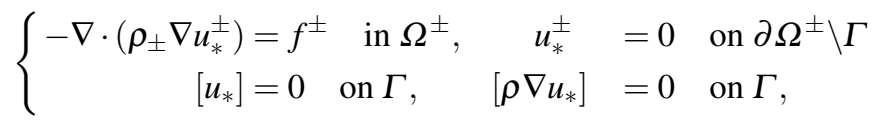

where $u_{*}^{ \pm}=\left.u_{*}\right|_{\Omega^{ \pm}}$and $\mathbf{n}^{ \pm}$denote the unit normal outward to $\Omega^{ \pm}$. The jump conditions on $\Gamma$ enforce the continuity of the solution and its flux across the interface.

Blanca Ayuso de Dios

Dipartimento di Matematica e Applicazioni, Università di Milano-Bicocca, Milan, Italy, e-mail: blanca.ayusoeunimib.it

Kyle Dunn

Cold Regions Research and Engineering Laboratory, ERDC - U.S. Army, Hanover, NH, e-mail: Kyle.G.Dunn@usace.army.mil

Marcus Sarkis

Mathematical Sciences Department, Worcester Polytechnic Institute, MA, e-mail: msarkis@ wpi.edu

Simone Scacchi

Dipartimento di Matematica, Università degli Studi di Milano, Milan, Italy, e-mail: simone. scacchi@unimi.it 
The jump operators are defined by

$$
\left[\rho \nabla u_{*}\right]=\rho_{+} \nabla u_{*}^{+} \cdot \mathbf{n}^{+}+\rho_{-} \nabla u_{*}^{-} \cdot \mathbf{n}^{-} \quad \text { and } \quad\left[u_{*}\right]=u_{*}^{+}-u_{*}^{-} .
$$

We also assume that the diffusion coefficients $\rho_{ \pm}>0$ are constant and satisfy $\rho_{-} \leq \rho_{+}$. Note that $u_{*}^{ \pm} \in H^{2}\left(\Omega^{ \pm}\right)$, but $u_{*} \in H^{1+\varepsilon}(\Omega)$ with $\varepsilon>0$. To approximate (1) we consider the stabilised unfitted FE approximation from [Burman et al., 2018].

A class of unfitted finite element methods were introduced in the seminal works of [Barrett and Elliott, 1987] and in recent years there has been a renewed interest in these type of approaches, giving rise to numerous novel methods; the immersed boundary method [Boffi and Gastaldi, 2003], XFEM [Chessa et al., 2002], the finite cell method (FCM) [Dauge et al., 2015], and CutFEM [Burman and Hansbo, 2012, Hansbo and Hansbo, 2002]. The use of unfitted meshes is particularly relevant for interface problems. However, in spite of the upsurge in research for unfitted approaches, the design and analysis of robust solvers for the resulting linear and nonlinear systems still seem elusive. Simple preconditioning strategies are explored for finite cell discretizations in [de Prenter et al., 2017] and multigrid-type method are proposed in [Ludescher et al., 2018]. In the present contribution we focus on the construction of a simple Dirichlet-Neumann (DN) domain decomposition preconditioner for the CutFEM method introduced in [Burman et al., 2018] and demonstrate its robustness also in the hard inclusion case. Due to space restrictions, we focus on a very simple version and stick to the algebraic description of the solver. Details on the analysis as well as further tailored preconditioners will be found in [Ayuso de Dios et al., 2019].

\section{Basic Notation and Unfitted Stabilized Discretization}

Let $\left\{\mathscr{T}_{h}\right\}_{h>0}$ be a family of uniform partitions of $\Omega$ into squares $T$ of diameter $h$. We assume that for each $T, \Gamma \cap \partial T$, is either empty or occurs at exactly two different edges of $\partial T^{1}$. We also define:

$$
\mathscr{T}_{h}^{ \pm}:=\left\{T \in \mathscr{T}_{h}: \bar{T} \cap \bar{\Omega}^{ \pm} \neq \emptyset\right\}, \quad \mathscr{T}_{h}^{\Gamma}:=\left\{T \in \mathscr{T}_{h}: \bar{T} \cap \Gamma \neq \emptyset\right\} .
$$

For $T \in \mathscr{T}_{h}^{\Gamma}$ we denote $T_{\Gamma}=\bar{T} \cap \Gamma$. We also introduce the discrete domains

$$
\Omega_{h}^{ \pm}:=\operatorname{Int}\left(\bigcup_{T \in \mathscr{T}_{h}^{ \pm}} \bar{T}\right) \quad \Omega_{h}^{\Gamma}:=\operatorname{Int}\left(\bigcup_{T \in \mathscr{T}_{h}^{\Gamma}} \bar{T}\right), \quad \text { and } \quad \Omega_{h, 0}^{ \pm}=\Omega_{h}^{ \pm} \backslash \bar{\Omega}_{h}^{\Gamma},
$$

where $\operatorname{Int}(K)$ denotes the interior of the set $K$. Note that $\Omega_{h}^{+} \cup \Omega_{h}^{-}=\Omega$ is an overlapping partition of $\Omega$ while a non-overlapping partition is given by $\Omega=$

${ }^{1}$ This assumption is only needed in the stability and error analysis of the method. 
$\Omega_{h, 0}^{+} \cup \bar{\Omega}_{h}^{\Gamma} \cup \Omega_{h, 0}^{-}$(see Figure 1.) Finally we introduce the following subsets of edges of elements in $\mathscr{T}_{h}^{\Gamma}$ :

$$
\mathscr{E}_{h}^{\Gamma, \pm}:=\left\{e=\operatorname{Int}\left(\partial T_{1} \cap \partial T_{2}\right): T_{1} \neq T_{2} \in \mathscr{T}_{h}^{ \pm}, \text {and } T_{1} \in \mathscr{T}_{h}^{\Gamma} \text { or/and } T_{2} \in \mathscr{T}_{h}^{\Gamma}\right\} .
$$

Note that $\mathscr{E}_{h}{ }^{,+}$(resp. $\mathscr{E}_{h}{ }^{,-}$) does not contain any edges on $\partial \Omega_{h}^{+}$(resp. $\partial \Omega_{h}^{-}$).
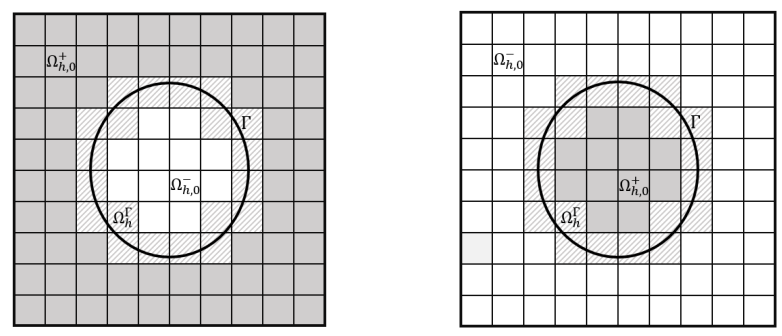

Fig. 1: Domain configurations:

Soft inclusion (leftmost),

Hard inclusion (center) cases

- Finite Element Spaces: We consider FE spaces of piecewise bilinear polynomials whose support is contained in $\Omega_{h}^{ \pm}, \Omega_{h, 0}^{ \pm}$and $\Omega_{h}^{\Gamma}$, respectively:

$$
\begin{gathered}
V^{ \pm}=\left\{v \in \mathscr{C}\left(\Omega_{h}^{ \pm}\right):\left.v\right|_{T} \in \mathbb{Q}^{1}(T), \forall T \in \mathscr{T}_{h}^{ \pm}, \text {and }\left.v\right|_{\partial \Omega_{h}^{ \pm} \cap \partial \Omega} \equiv 0\right\}, \\
V_{0}^{ \pm}=\left\{v \in V^{ \pm}:\left.v\right|_{T} \equiv 0 \quad \forall T \in \Omega_{h}^{\Gamma}\right\}, \quad W^{ \pm}=\left\{v \text { restricted to } \Omega_{h}^{\Gamma}, \quad v \in V^{ \pm}\right\} .
\end{gathered}
$$

With a small abuse of notation, we set $V_{h}=V^{+} \times V^{-}$where it is understood

$$
u_{h} \in V_{h}=V^{+} \times V^{-} \quad u_{h}=\left(u^{+}, u^{-}\right) \text {with } u^{+} \in V^{+}, \quad u^{-} \in V^{-} .
$$

That is, the FE space $V_{h}$ is defined by a copy of two FE piecewise functions: one from $V^{+}$defined on $\Omega_{h}^{+}$and another from $V^{-}$defined over $\Omega_{h}^{-}$.

- The stabilised unfitted Nitsche approximation: the method reads: find $u_{h}=$ $\left(u^{+}, u^{-}\right) \in V_{h}=V^{+} \times V^{-}$, such that:

$$
a_{h}\left(u_{h}, v_{h}\right)=\left(f^{+}, v^{+}\right)_{\Omega^{+}}+\left(f^{-}, v^{-}\right)_{\Omega^{-}}, \quad \text { for all } v_{h}=\left(v^{+}, v^{-}\right) \in V^{+} \times V^{-},
$$

where $(\cdot, \cdot)_{\Omega^{ \pm}}$denotes the $L^{2}\left(\Omega^{ \pm}\right)$inner product and $a_{h}: V_{h} \times V_{h} \longrightarrow \mathbb{R}$ is given as:

$$
\begin{aligned}
& a_{h}\left(u_{h}, v_{h}\right)=\int_{\Omega^{-}} \rho_{-} \nabla u^{-} \cdot \nabla v^{-} d x+\int_{\Omega^{+}} \rho_{+} \nabla u^{+} \cdot \nabla v^{+} d x \\
& +\int_{\Gamma}\left(\left\{\rho \nabla v_{h}\right\}_{w} \cdot \mathbf{n}^{-}\left[u_{h}\right]+\left\{\rho \nabla u_{h}\right\}_{w} \cdot \mathbf{n}^{-}\left[v_{h}\right]\right) d s+\sum_{T \in \mathscr{T}_{h}^{\Gamma}} \frac{\gamma_{\Gamma}}{h_{T}}\{\rho\}_{H} \int_{T_{\Gamma}}\left[u_{h}\right]\left[v_{h}\right] d s \\
& +\sum_{e \in \mathscr{E}_{h},-} \gamma_{-}|e| \int_{e} \rho_{-}\left[\nabla u^{-}\right]\left[\nabla v^{-}\right] d s+\sum_{e \in \mathscr{E}_{h},+} \gamma_{+}|e| \int_{e} \rho_{+}\left[\nabla u^{+}\right]\left[\nabla v^{+}\right] d s,
\end{aligned}
$$


where $\gamma^{\Gamma}, \gamma^{-}$, and $\gamma^{+}$are positive (moderate) constants and $|e|$ is the diameter of the edge $e$. Here, $[\cdot]$ refers to the jump operator as in (2) while $\{\cdot\}_{H}$ and $\{\cdot\}_{\omega}$ denote the harmonic and weighted averages defined by:

$\{\rho\}_{H}=\frac{2 \rho^{+} \rho^{-}}{\rho^{+}+\rho^{-}}, \quad\left\{\rho \nabla v_{h}\right\}_{\omega}:=\left(\omega_{-} \rho^{-} \nabla v^{-}+\omega_{+} \rho^{+} \nabla v^{+}\right), \quad \omega_{\mp}=\frac{\rho^{ \pm}}{\rho^{+}+\rho^{-}}$.

Continuity and coercivity of $a_{h}(\cdot, \cdot)$ in (4) can be shown with respect to the norm:

$$
\begin{aligned}
& \left\|v_{h}\right\|_{V_{h}}^{2}:=\left|v^{+}\right|_{V^{+}}^{2}+\left|v^{-}\right|_{V^{-}}^{2}+\sum_{T \in \mathscr{T}_{h}^{\Gamma}} \frac{\gamma_{\Gamma}}{h_{T}}\{\rho\}_{H} \int_{T_{\Gamma}}\left[v_{h}\right]^{2} d s \quad \forall v_{h} \in V_{h}, \quad \text { with } \\
& \left|v^{ \pm}\right|_{V^{ \pm}}^{2}:=\int_{\Omega^{ \pm}} \rho_{ \pm}\left|\nabla v^{ \pm}\right|^{2} d x+\sum_{e \in \mathscr{E}_{h}^{\Gamma, \pm}} \gamma_{ \pm}|e| \int_{e} \rho_{ \pm}\left[\nabla v^{ \pm}\right]^{2} d s, \quad \forall v^{ \pm} \in V^{ \pm} .
\end{aligned}
$$

We remark that the semi-norm $|\cdot|_{V^{+}}$is a norm if $\Omega^{+}$is non floating. We will denote by $(\cdot, \cdot)_{V^{+}}$to its originating inner product. Optimal and robust error estimates are proved in [Burman et al., 2018].

\section{Dirchlet-Neumann preconditioner}

We describe now a preconditioner for the linear system resulting from (3) based on the non-overlapping decomposition $\Omega_{h, 0}^{+} \cup \bar{\Omega}_{h}^{\Gamma} \cup \Omega_{h, 0}^{-}$. Associated with such a decomposition, and owing to the fat interface we consider the somehow asymmetric splitting of the space $V_{h}=\left(V_{0}^{+}, W^{+}\right) \times V^{-}$, we first introduce some notation. We denote by $\mathscr{R}_{ \pm}: V_{h} \longrightarrow V^{ \pm}$the restriction operators to $\Omega_{h}^{ \pm}$such that $\mathscr{R}_{ \pm} u_{h}=u^{ \pm}$. The corresponding prolongation operators $\mathscr{R}_{ \pm}^{T}: V^{ \pm} \longrightarrow V_{h}$ are defined as the extension to $V_{h}$ by zero, i.e., $\mathscr{R}_{+}^{T} u^{+}=\left(u^{+}, 0\right)$ and $\mathscr{R}_{-}^{T} u^{-}=\left(0, u^{-}\right)$. Similarly, we introduce the restriction and prolongation operators

$$
\begin{array}{lll}
\mathscr{R}_{W^{ \pm}}: V_{h} \longrightarrow W^{ \pm} & \mathscr{R}_{0^{ \pm}}: V_{h} \longrightarrow V_{0}^{ \pm} & \mathscr{R}_{W}: V_{h} \longrightarrow W_{h} \\
\mathscr{R}_{W^{ \pm}}^{T}: W^{ \pm} \longrightarrow V_{h} & \mathscr{R}_{0^{ \pm}}^{T}: V_{0}^{ \pm} \longrightarrow V_{h} & \mathscr{R}_{W}^{T}: W_{h} \longrightarrow V_{h}
\end{array}
$$

We define the bilinear forms $a_{0}^{+}: V_{0}^{+} \times V_{0}^{+} \longrightarrow \mathbb{R}$ and $a^{-}: V^{-} \times V^{-} \longrightarrow \mathbb{R}$

$$
\begin{array}{lll}
a_{0}^{+}: V_{0}^{+} \times V_{0}^{+} \longrightarrow \mathbb{R} & a_{0}^{+}\left(u_{0}^{+}, v_{0}^{+}\right):=a_{h}\left(\mathscr{R}_{0^{+}}^{T} u_{0}^{+}, \mathscr{R}_{0^{+}}^{T} v_{0}^{+}\right) & \forall u_{0}^{+}, v_{0}^{+} \in V_{0}^{+} \\
a^{-}: V^{-} \times V^{-} \longrightarrow \mathbb{R} & a^{-}\left(u^{-}, v^{-}\right):=a_{h}\left(\mathscr{R}_{-}^{T} u^{-}, \mathscr{R}_{-}^{T} v^{-}\right) & \forall u^{-}, v^{-} \in V^{-}
\end{array}
$$

We now introduce the local solvers. Let $u_{f, 0}^{+} \in V_{0}^{+}$and $u_{f}^{-} \in V^{-}$be the local solutions with support in $\Omega_{h, 0}^{+}$and $\Omega_{h}^{-}$, respectively, defined by:

$$
a_{0}^{+}\left(u_{f, 0}^{+}, v_{0}^{+}\right)=\left(f^{+}, v_{0}^{+}\right)_{\Omega^{+}} \forall v_{0}^{+} \in V_{0}^{+} \quad a^{-}\left(u_{f}^{-}, v^{-}\right)=\left(f^{-}, v^{-}\right)_{\Omega^{-}} \quad v^{-} \in V^{-} .
$$


We set $\mathscr{P}_{h} u_{h}=\mathscr{R}_{0^{+}}^{T} u_{f, 0}^{+}+\mathscr{R}_{-}^{T} u_{f}^{-}$and note that $u_{h}-\mathscr{P}_{h} u_{h}$ lies in the orthogonal complement of $\mathscr{R}_{0^{+}}^{T} V_{0}^{+}+\mathscr{R}_{-}^{T} V^{-}$in $V_{h}$ with respect to the inner product $a_{h}(\cdot, \cdot)$. This suggests the splitting $u_{h}=\mathscr{P}_{h} u_{h}+\mathscr{H}_{h} u_{h}$, with $\mathscr{H}_{h} u_{h}=\left(\mathscr{H}_{+} u_{h}, \mathscr{H}_{-} u_{h}\right) \in V_{h}$ a suitable discrete harmonic extension of $\left.\left(u_{h}^{+}\right)\right|_{\Omega^{\Gamma}}$ that we briefly sketch next. Recall that $W^{+}$is the restriction of the space $V^{+}$to $\Omega_{h}^{\Gamma}$. Given $\eta^{+} \in W^{+}$, we define $\mathscr{H}_{ \pm}$: $W^{+} \longrightarrow V^{ \pm}$to be the discrete harmonic extension of $\eta^{+}$such that

$$
a_{h}\left(\mathscr{R}_{+}^{T} \mathscr{H}_{+} \eta^{+}, \mathscr{R}_{0^{+}}^{T} v_{0}^{+}\right)=0 \quad \forall v_{0}^{+} \in V_{0}^{+} \quad \text { and } \quad \mathscr{R}_{W^{+}} \mathscr{R}_{+}^{T} \mathscr{H}_{+} \eta^{+}=\eta^{+}
$$

and

$$
a_{h}\left(\left(\mathscr{R}_{+} \mathscr{R}_{W^{+}}^{T} \eta^{+}, \mathscr{H}_{-} \eta^{+}\right), \mathscr{R}_{-}^{T} v^{-}\right)=0 \quad \forall v^{+} \in V^{-} .
$$

Finally, we set $\mathscr{H}_{h} \eta^{+}=\left(\mathscr{H}_{+} \eta^{+}, \mathscr{H}_{-} \eta^{+}\right)$and introduce the Schur complement operator $\mathscr{S}: W^{+} \longrightarrow W^{+}$:

$$
<\mathscr{S} \eta, w>:=a_{h}\left(\mathscr{H}_{h} \eta^{+}, \mathscr{H}_{h} w^{+}\right) \quad \forall \eta^{+}, w^{+} \in W^{+} .
$$

From the definition of $\mathscr{P}_{h} u_{h}$ it follows

$$
a_{h}\left(\mathscr{H}_{h} u_{h}, \mathscr{H}_{h} v_{h}\right)=\left(f, v_{h}\right)_{\Omega}-a_{h}\left(\mathscr{P}_{h} u_{h}, v_{h}\right) \quad \forall v_{h} \in V_{h} .
$$

We focus now on constructing preconditioners $\mathscr{B}^{-1}$ for the operator $\mathscr{S}$ and hence for the system (7). The basic guide to ensure robustness will be to use, when possible, the local Schur complement corresponding to the largest coefficient, $\rho_{+}$:

$$
<\mathscr{S}_{+} \eta, w>:=\left(\mathscr{H}_{+} \eta, \mathscr{H}_{+} w^{+}\right)_{V^{+}} \quad \forall \eta, w \in W^{+},
$$

where $(\cdot, \cdot)_{V+}$ is the originating inner-product for the norm $|\cdot|_{V^{+}}$in (5). We need to distinguish two cases:

- $\Omega^{+}$is not floating subdomain and we set $\mathscr{B}^{-1}=\mathscr{S}_{+}^{-1}$.

- $\Omega^{+}$is a floating subdomain; since $\mathscr{S}_{+}$is not invertible, we define $\mathscr{B}^{-1}$ as a suitable regularisation of $\mathscr{S}_{+}$. We propose one level and two level methods.

\section{Algebraic formulation of the DN preconditioner}

After choosing standard Lagrangian basis for $V^{ \pm}$, problem (3) reduces to a linear algebraic system $\mathbb{A} \mathbb{U}=\mathbb{F}$. We consider the block structure of $\mathbb{A}$ that results from splitting the degrees of freedom (dofs) of the discrete space $V_{h}$ into three sets:

- dofs associated with $V_{0}^{+}$(in the interior of $\Omega^{+}$) are indicated by $I^{+}$;

- dofs related to $W^{+}$, indicated by $W^{+}$;

- dofs associated with $V^{-}$(dofs related to $V_{0}^{-}$and $\left.W^{-}\right)$, indicated by $V^{-}$. 


$$
\left[\begin{array}{ccc}
\mathbb{A}_{I^{+} I^{+}} & \mathbb{A}_{I^{+} W^{+}} & 0 \\
\mathbb{A}_{W^{+} I^{+}} & \mathbb{A}_{W^{+} W^{+}}^{+}+\mathbb{A}_{W^{+} W^{+}}^{-} & \mathbb{A}_{W^{+} V^{-}} \\
\cline { 2 - 2 } & \multicolumn{1}{c}{\mathbb{A}_{V^{-} W^{+}}} & \mathbb{A}_{V^{-} V^{-}}
\end{array}\right]\left[\begin{array}{c}
\mathbb{U}_{I^{+}} \\
\mathbb{U}_{W^{+}} \\
\mathbb{U}_{V^{-}}
\end{array}\right]=\left[\begin{array}{l}
\mathbb{F}_{I^{+}} \\
\mathbb{F}_{W^{+}} \\
\mathbb{F}_{V^{-}}
\end{array}\right] .
$$

Here, we have highlighted that the stiffness block with dofs from $W^{+}$in the fat interface has contributions from $\Omega_{h}^{+}$and $\Omega_{h}^{-}$. Performing static condensation of the interior variables $I^{+}$and $V^{-}$we obtain the Schur complement system

$$
\mathbb{S U}_{W^{+}}=\mathbb{G}_{W^{+}}, \quad \mathbb{S}=\mathbb{S}_{+}+\mathbb{S}_{-},
$$

where $\mathbb{G}_{W^{+}}=\mathbb{F}_{W^{+}}-\mathbb{A}_{W^{+} I^{+}} \mathbb{A}_{I^{+} I^{+}}^{-1} \mathbb{F}_{I^{+}}-\mathbb{A}_{W^{+} V^{-}} \mathbb{A}_{V^{-} V^{-}}^{-1} \mathbb{F}_{V^{-}}$, and $\mathbb{S}$ is given by

$$
\mathbb{S}=\mathbb{S}_{+}+\mathbb{S}_{-} \quad \text { with } \quad\left\{\begin{array}{l}
\mathbb{S}_{+}=\mathbb{A}_{W^{+} W^{+}}^{+}-\mathbb{A}_{W^{+} I^{+}} \mathbb{A}_{I^{+} I^{+}}^{-1} \mathbb{A}_{I^{+} W^{+}} \\
\mathbb{S}_{-}=\mathbb{A}_{W^{+} W^{+}}^{-}-\mathbb{A}_{W^{+} V^{-}} \mathbb{A}_{V^{-} V^{-}}^{-} \mathbb{A}_{V^{-} W^{+}}
\end{array}\right.
$$

Soft inclusion: $\Omega_{h}^{+}$in Non-Floating Subdomain Case: In this case we set $\mathscr{B}^{-1}=\mathscr{S}_{+}^{-1}$ since the operator is invertible. At the algebraic level we arrive at $\mathbb{S}_{+}^{-1} \mathbb{S U}_{W^{+}}=\mathbb{S}_{+}^{-1} \mathbb{G}_{W^{+}}$. The action of the DN preconditioner $\mathbb{S}_{+}^{-1}$ on a generic residual vector $\mathbb{R}_{W^{+}}$consists of solving the linear system

$$
\left[\begin{array}{ll}
\mathbb{A}_{I^{+} I^{+}} & \mathbb{A}_{I^{+} W^{+}} \\
\mathbb{A}_{W^{+} I^{+}} & \mathbb{W}_{W^{+} W^{+}}^{+}
\end{array}\right]\left[\begin{array}{l}
\mathbb{V}_{I^{+}} \\
\mathbb{V}_{W^{+}}
\end{array}\right]=\left[\begin{array}{l}
0 \\
\mathbb{R}_{W^{+}}
\end{array}\right]
$$

and letting $\mathbb{V}_{W^{+}}:=\mathbb{S}_{+}^{-1} \mathbb{R}_{W^{+}}$.

Hard inclusion: $\Omega_{h}^{+}$is the Floating Subdomain: Since $\mathbb{S}_{+}$is not invertible we consider two different strategies: a regularisation and the use of a one dimensional coarse solver to account for the kernel of $\mathbb{S}_{+}$.

- One-Level DN: The action of the preconditioner amounts to solving

$$
\left(\left[\begin{array}{cc}
\mathbb{A}_{I^{+} I^{+}} & \mathbb{A}_{I^{+} W^{+}} \\
\mathbb{A}_{W^{+} I^{+}} & \mathbb{A}_{W^{+} W^{+}}^{+}
\end{array}\right]+\frac{\{\rho\}_{H}}{D_{+}^{2}}\left[\begin{array}{cc}
\mathbb{M}_{I^{+} I^{+}}^{+} & \mathbb{M}_{I^{+} W^{+}}^{+} \\
\mathbb{M}_{W^{+} I^{+}}^{+} & \mathbb{M}_{W^{+} W^{+}}^{+}
\end{array}\right]\right)\left[\begin{array}{l}
\mathbb{V}_{I^{+}} \\
\mathbb{V}_{W^{+}}
\end{array}\right]=\left[\begin{array}{l}
0 \\
\mathbb{R}_{W^{+}}
\end{array}\right],
$$

and setting $\mathbb{S}_{+, \text {one }}^{-1} \mathbb{R}_{W^{+}}=\mathbb{V}_{W^{+}}$. Here, $\mathbb{M}^{+}$stands for the mass matrix associated with $V^{+}$(i.e., defined over $\Omega_{h}^{+}$), and $D_{+}:=\operatorname{diam}\left(\Omega_{h}^{+}\right)$and is used to regularise $\mathbb{S}_{+}$.

- Two Level DN preconditioner: The idea is to first solve in the space orthogonal to the (one-dimensional) kernel of $\mathbb{S}_{+}$and then correct with a coarse solver that accounts for the contribution in $\operatorname{ker}\left(\mathbb{S}_{+}\right)$. Hence, the practical implementation of the two level solver $\mathbb{S}_{+, t w o}^{-1}$ amounts to first solving

$$
\left[\begin{array}{cc}
\mathbb{A}_{I^{+} I^{+}} & \mathbb{A}_{I^{+} W^{+}} \\
\mathbb{A}_{W^{+} I^{+}} & \mathbb{A}_{W^{+} W^{+}}^{+}
\end{array}\right]\left[\begin{array}{l}
\mathbb{V}_{I^{+}} \\
\mathbb{V}_{W^{+}}
\end{array}\right]+\left[\begin{array}{cc}
\mathbb{M}_{I^{+} I^{+}}^{+} & \mathbb{M}_{I^{+} W^{+}}^{+} \\
\mathbb{M}_{W^{+} I^{+}}^{+} & \mathbb{M}_{W^{+} W^{+}}^{+}
\end{array}\right]\left[\begin{array}{l}
\mathbf{1}_{I^{+}} \\
\mathbf{1}_{W^{+}}
\end{array}\right] \lambda=\left[\begin{array}{l}
0 \\
\mathbb{R}_{W^{+}}
\end{array}\right],
$$

with the constraint 


$$
\left[\begin{array}{l}
\mathbf{1}_{I^{+}} \\
\mathbf{1}_{W^{+}}
\end{array}\right]^{T}\left[\begin{array}{cc}
\mathbb{M}_{I^{+}+}^{+} & \mathbb{M}_{I^{+} W^{+}}^{+} \\
\mathbb{M}_{W^{+} I^{+}}^{+} & \mathbb{M}_{W^{+} W^{+}}^{+}
\end{array}\right]\left[\begin{array}{c}
\mathbb{V}_{I^{+}} \\
\mathbb{V}_{W^{+}}
\end{array}\right]=0
$$

and then define $\mathbb{S}_{+}^{\dagger} \mathbb{R}_{W^{+}}=\mathbb{V}_{W^{+}}$. Here $\mathbf{1}_{I^{+}}^{+}$and $\mathbf{1}_{W^{+}}^{+}$are vectors of ones in $V_{0}^{+}$ and $W^{+}$, respectively. The matrix representation of the two level preconditioner (with coarse space) is defined via $\mathbb{S}_{+, t w o}^{-1}=\mathbb{S}_{+}^{\dagger}+\mathbf{1}_{W^{+}}\left(\mathbf{1}_{W^{+}}, \mathbb{S}_{W^{+}}\right)^{-1} \mathbf{1}_{W^{+}}^{T}$. Note that $\left.\mathbb{S 1}_{W^{+}}=\mathbb{S}_{-} \mathbf{1}_{W^{+}}\right)$.

\section{Numerical Results}

We consider the domain $\Omega=[0,1]^{2}$ and study the performance of the DirichletNeumann (DN) preconditioner for the CutFEM approximation (3) to (1) with $\Omega^{\mp}$ a disk of radius 0.15 and $\Omega^{ \pm}=(0,1)^{2} \backslash \bar{\Omega}^{\mp}$ and always $\rho_{-} \leq \rho_{+}$. We use CG and PCG as a solver with zero initial guess and tolerance $10^{-6}$ for the relative residual. In the tables we report the estimated (via Lanzcos algorithm) condition numbers (denoted by $\kappa_{2}$ ) and the number of iterations (denoted by it) required by CG and PCG for convergence. Table 1 reports the results in the case where $\Omega^{+}$is non-floating,

\begin{tabular}{||c|cc|cc|cc||}
\hline$\rho_{-}$ & \multicolumn{2}{|c|}{ full CG } & \multicolumn{3}{c||}{ schur NO precond. } & \multicolumn{3}{c||}{ schur DN preconditioned } \\
\hline & $\kappa_{2}$ & it & $\kappa_{2}$ & it & $\kappa_{2}$ & it \\
\hline 1 & $3.32 \mathrm{e}+3$ & $(218)$ & 388.40 & $(75)$ & 1.95 & $(14)$ \\
$10^{-2}$ & $2.06 \mathrm{e}+4$ & $(575)$ & 362.15 & $(91)$ & 1.01 & $(15)$ \\
$10^{-4}$ & $2.01 \mathrm{e}+6$ & $(2828)$ & 361.71 & $(93)$ & 1.00 & $(4)$ \\
$10^{-6}$ & $2.01 \mathrm{e}+8$ & $(5418)$ & 361.70 & $(93)$ & 1.00 & $(3)$ \\
\hline
\end{tabular}

Table 1: Robustness with respect to $\rho: \Omega^{-}$is the floating subdomain. Here, $\rho_{+}=1$ and $h=1 / 64$.

therefore using $\mathscr{S}_{+}^{-1}$ as a preconditioner. $\mathscr{S}_{+}^{-1}$ performs robustly when the ratio $\rho_{+} / \rho_{-}$increases. In the case where $\Omega^{+}$is the floating subdomain, we use one level and two level DN preconditioners. The results regarding optimality and robustness of these preconditioners are reported in Table 2 and 3, respectively. Notice that both preconditioners perform optimally and show robustness with respect to the jumping coefficient. In particular, the one-level DN preconditioner seems to be enough effective for the considered setting.

\section{References}

[Ayuso de Dios et al., 2019] Ayuso de Dios, B., Dunn, K., Sarkis, M., and Scacchi, S. (2019). Simple preconditioners for cutfem methods. (work in preparation).

[Barrett and Elliott, 1987] Barrett, J. W. and Elliott, C. M. (1987). Fitted and unfitted finiteelement methods for elliptic equations with smooth interfaces. IMA J. Numer. Anal., 7(3):283300 . 


\begin{tabular}{||c|cc|cc|cc|cc||}
\hline $1 / h$ & \multicolumn{2}{|c|}{ full CG } & \multicolumn{2}{c|}{ schur NO precond. } & \multicolumn{2}{c|}{ DN Two-Level } & \multicolumn{2}{c|}{ DN one-level } \\
& $\kappa_{2}$ & it & $\kappa_{2}$ & it & $\kappa_{2}$ & it & $\kappa_{2}$ & it \\
\hline 8 & $6.38 \mathrm{e}+3$ & 252 & $4.09 \mathrm{e}+2$ & 79 & 6.76 & 11 & 3.51 & 14 \\
16 & $1.77 \mathrm{e}+4$ & 520 & $8.60 \mathrm{e}+3$ & 224 & 6.39 & 15 & 2.11 & 14 \\
32 & $5.83 \mathrm{e}+4$ & 863 & $1.09 \mathrm{e}+4$ & 423 & 6.29 & 16 & 2.09 & 14 \\
64 & $2.14 \mathrm{e}+4$ & 1625 & $1.86 \mathrm{e}+4$ & 551 & 6.34 & 16 & 2.08 & 14 \\
128 & $8.19 \mathrm{e}+5$ & 3163 & $3.79 \mathrm{e}+4$ & 832 & 6.37 & 16 & 2.13 & 14 \\
256 & $3.20 \mathrm{e}+6$ & 6140 & $7.43 \mathrm{e}+4$ & 1148 & 6.39 & 16 & 2.19 & 14 \\
\hline
\end{tabular}

Table 2: Optimality with respect to $h$ : floating circle $\Omega^{+}$embedded in $[0,1]^{2} \cdot \rho_{+}=\rho_{-}=1$.

\begin{tabular}{||c|cc|cc|cc|cc||}
\hline$\rho_{+}$ & \multicolumn{2}{|c|}{ full CG } & \multicolumn{2}{c|}{ schur NO precond. } & \multicolumn{2}{c|}{ DN Two-Level } & \multicolumn{2}{c|}{ DN one-level } \\
& $\kappa_{2}$ & it & $\kappa_{2}$ & it & $\kappa_{2}$ & it & $\kappa_{2}$ & it \\
\hline 1 & $2.14 \mathrm{e}+5$ & 1625 & $1.86 \mathrm{e}+4$ & 539 & 6.37 & 16 & 2.13 & 14 \\
$10^{2}$ & $2.00 \mathrm{e}+7$ & 12906 & $1.81 \mathrm{e}+6$ & 765 & 6.33 & 6 & 1.83 & 5 \\
$10^{4}$ & $2.00 \mathrm{e}+9$ & $>100000$ & $1.81 \mathrm{e}+8$ & 897 & 6.33 & 4 & 1.83 & 4 \\
$10^{6}$ & $5.70 \mathrm{e}+10$ & $>100000$ & $1.81 \mathrm{e}+10$ & 1026 & 6.33 & 3 & 1.83 & 3 \\
$10^{8}$ & $4.20 \mathrm{e}+12$ & $>100000$ & $1.83 \mathrm{e}+12$ & 1326 & 6.33 & 3 & 1.83 & 3 \\
\hline
\end{tabular}

Table 3: Robustness with respect to $\rho$. Floating $\Omega^{+}$with jumping coefficients. Here, $\rho_{-}=1$, $1 / h=64$.

[Boffi and Gastaldi, 2003] Boffi, D. and Gastaldi, L. (2003). A finite element approach for the immersed boundary method. Comput. \& Structures, 81(8-11):491-501. In honour of KlausJürgen Bathe.

[Burman et al., 2018] Burman, E., Guzmán, J., Sánchez, M. A., and Sarkis, M. (2018). Robust flux error estimation of an unfitted Nitsche method for high-contrast interface problems. IMA J. Numer. Anal., 38(2):646-668.

[Burman and Hansbo, 2012] Burman, E. and Hansbo, P. (2012). Fictitious domain finite element methods using cut elements: II. A stabilized Nitsche method. Appl. Numer. Math., 62(4):328341.

[Chessa et al., 2002] Chessa, J., Smolinski, P., and Belytschko, T. (2002). The extended finite element method (XFEM) for solidification problems. Internat. J. Numer. Methods Engrg., 53(8):1959-1977.

[Dauge et al., 2015] Dauge, M., Düster, A., and Rank, E. (2015). Theoretical and numerical investigation of the finite cell method. J. Sci. Comput., 65(3):1039-1064.

[de Prenter et al., 2017] de Prenter, F., Verhoosel, C. V., van Zwieten, G. J., and van Brummelen, E. H. (2017). Condition number analysis and preconditioning of the finite cell method. Comput. Methods Appl. Mech. Engrg., 316:297-327.

[Hansbo and Hansbo, 2002] Hansbo, A. and Hansbo, P. (2002). An unfitted finite element method, based on Nitsche's method, for elliptic interface problems. Comput. Methods Appl. Mech. Engrg., 191(47-48):5537-5552.

[Ludescher et al., 2018] Ludescher, T., Gro $\beta$, S., and Reusken, A. (2018). A multigrid method for unfitted finite element discretizations of elliptic interface problems. Techical report IGPM 481, RWTH Aachen. 